\title{
FORMACIÓN DOCENTE EN LA RESIDENCIA. ¿EXPERIENCIA SUBJETIVANTE?
}

\author{
Teacher Training in the Residence Program. Experience for subjectivizing?
}

María Soledad Manrique ${ }^{1}$

soledadmanrique@conicet.gov.ar

\begin{abstract}
${ }^{1}$ Consejo Nacional de Investigaciones Científicas y Técnicas. Argentina
\end{abstract}

Fecha de recepción: 28/02/2020

Fecha de aprobación: 9/06/2020

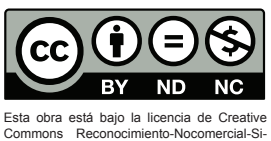

\section{Resumen}

A partir de la pregunta acerca de las oportunidades que brinda la instancia de residencia docente para el desarrollo de la subjetividad y del sí mismo profesional, el trabajo aborda la descripción y análisis del recorrido de dos futuras docentes de nivel de primario por la residencia a lo largo de un semestre. Con las teorías de la complejidad como marco epistemológico, el objeto se aborda metodológicamente desde el enfoque clínico, que se vale del estudio de caso de dos docentes en formación. El seguimiento involucró registros de campo de situaciones vividas durante la residencia en las escuelas y entrevistas en profundidad. El análisis de contenido, el análisis del discurso y el análisis multirreferenciado se complementaron con análisis clínicos en profundidad. Los resultados mostraron cómo el deseo de enseńar de las docentes en formación fue puesto a prueba y sufrió transformaciones en la medida en que sus mecanismos de defensa entraron en juego en una situación compleja de demandas contradictorias. En el entramado entre su entorno y la particular forma de posicionarse y de responder al conflicto de las docentes consideradas toma forma una instancia de formación que brinda oportunidades para la subjetivación o para la desubjetivación, para la construcción o no de una experiencia, para el agenciamiento o la inhibición de la propia actividad deseante. Varios conceptos de un marco teórico psicoanalítico y foucaultiano complementan el análisis y permiten ponderar la relevancia de la residencia en su calidad de instancia de construcción de subjetividad.

Palabras clave: estudiante, profesor, formación de docentes, práctica pedagógica, psicoanálisis, psicología de la educación, supervisión de los docentes.

\section{Abstract}

Based on the question about the opportunities offered by the teaching residence for the development of subjectivity and the professional self, the work addresses the description and analysis of the passage through the residence program of two future primary teachers throughout a semester. With theories of complexity as an epistemological framework, the object is delt with methodologically from the clinical approach, which employs the case study of two teachers in training. Accompaniment of the two teachers involved field records of situations experienced during their residence in schools and indepth interviews. Content analysis, discourse analysis and multireferenced analysis were complemented with in-depth clinical analysis. The results showed how desire to teach was tested and underwent transformations in the two teachers and their defense mechanisms came into play in a complex situation of conflicting demands. In the intersection between their environment and the particular way of positioning themselves and responding to the conflict of the teachers considered, an instance of training takes place that provides opportunities for subjectivation or desubjectivation, for the construction or not of an experience, for the agency or inhibition of the activity of feeling desire itself. Concepts of a Psychoanalytic and Foucaultian theoretical framework complement the analysis and allow us to weigh the relevance of the residence program as an instance of construction of subjectivity.

Keywords: student, teachers, teacher education, psychoanalysis, educational psychology, teaching practice, teacher supervision. 


\section{Revista Caribeña de Investigación Educativa | 2021, 5(1), 73-85}

\section{Introducción}

La formación docente puede ser entendida desde una mirada sobre el sistema como un conjunto de acciones organizadas para poder ofrecer a futuros docentes la posibilidad de relacionarse con saberes y conocimientos. Puede ser entendida intersubjetivamente, como relación entre un sujeto en formación y sus formadores e instancias de formación. Por último, desde una mirada intrasubjetiva, la formación también puede conceptualizarse como transformación del propio sujeto que se va involucrando en acciones sociales nuevas, que, al transformarse, transforma su medio (Ferry, 1997).

La formación docente en cuanto conjunto de acciones organizadas, se orienta a anticipar los desempeños profesionales, es decir, trabaja en gran medida sobre el desarrollo de capacidades (Barbier, 1999) en situaciones simuladas que podrán convertirse en competencias en el ejercicio profesional. El campo de las prácticas en la formación docente se enfoca en el desarrollo de un saber hacer, una praxis, un conocimiento procedimental, una acción deliberada que se realiza en función de determinados fines y responde a valores en relación con un contexto. Así, formarse en una práctica implica siempre ingresar a una comunidad de prácticos que comparten tradiciones, convenciones, jergas, sistemas de valores (Schön, 1998). En este caso, la comunidad involucra los institutos de formación, las escuelas en las que se realizan las prácticas y todo el sistema escolar que los aloja. La práctica profesional supervisada, como la denomina Steiman (2018), supone aprender a decidir, ocupa una posición en este sistema de tareas, de lugares y jerarquías. Esta división, señala el autor, está reglada por las formas de organización del tiempo y del espacio escolares, y en ellas están implícitas las representaciones explicitas e implícitas de las propias prácticas profesionales supervisadas dentro del sistema y las que portan los actores mismos.

Dentro del campo de la práctica profesional supervisada, la residencia es la instancia formativa de mayor relevancia y envergadura de nuestro actual sistema de formación docente. Con ella se completa la formación en el cuarto año de estudio. Constituye lo que Souto (2016) denomina "un dispositivo complejo" que integra distintas instancias de formación, realizadas en el ámbito del instituto de formación y en la escuela primaria elegida como escuela destino para que los residentes realicen sus prácticas. En cuanto arreglo de tiempos, espacio, personas, componentes teóricos y técnicos orientados a metas, dispone ciertas cosas y pone a disposición otras. Se da en la temporalidad, en la duración y trabaja sobre los procesos (Souto, 1999). La residencia en cuanto dispositivo puede ser entendida como instancia de pasaje temporal — dura un tiempo limitado-; pasaje espacial entre la institución de formación y las de trabajo; pasaje entre culturas, normativas, tradiciones, códigos compartidos, de modalidades institucionales, organizativas; y pasaje entre dos posiciones: de la de alumno a la de docente (Souto, 2016).

Interesadas por la pregunta acerca de qué sentido cobra la residencia para quienes la atraviesan y cómo afecta el desarrollo de su subjetividad y de su sí mismo profesional (Abraham, 1987), en el marco del proyecto UBACyT 2014-2017 "La formación profesional: la residencia docente en el marco de la formación en las prácticas", hemos acompañado durante un semestre a dos residentes de profesorado de Primaria a quienes desde ahora llamaremos Francisca y Yolanda. El trabajo aborda la descripción y análisis de su experiencia durante la formación en la instancia de residencia, del modo en que lidian con las demandas que se les presentan y, particularmente, del destino que sufre en este tránsito su deseo de enseñar.

En el paper se presenta un resumen del análisis en profundidad realizado. Una serie de conceptos psicoanalíticos (Rodulfo, 2013; Bleichmar, 2005), de la psicodinámica del trabajo (Dejours, 2019) y foucaultianos (Foucault, 1991, 2000; Ramírez-Zuluaga, 2015) complementan nuestro análisis.

Esperamos con esta investigación contribuir al conocimiento de algunas de las posibilidades, así como de las limitaciones que brinda esta instancia formativa y de diferentes modos de vivenciarla. Consideramos que este conocimiento puede constituir un aporte 
fundamental a la hora de tomar decisiones en el futuro que contemplen la posibilidad de afectar el dispositivo de formación docente y la forma de llevarlo a cabo.

\section{Revisión de la literatura}

Hablar de la formación es aludir a una subjetividad que se forma. Desde la concepción de la complejidad, entendemos la subjetividad como una entidad polifónica, compleja, múltiplemente atravesada, dinámica, histórica y social (Ardoino, 2005; Najmanovich, 2011). Una entidad que se co-construye de modo no lineal en devenir permanente junto con su entorno, como efecto de mecanismos de subjetivación mientras, simultáneamente, construye el universo de sentido en que se desenvuelve y la actividad misma (Barbier, 2017). Una entidad, por fin, que en tanto se encuentra atravesada por el inconsciente, por el otro, por la cultura, por el lenguaje, no se autodetermina, sino que es con su entorno, pero que es capaz, también, en oportunidades, de tomar distancia de sí, y a través de ese trabajo de deconstrucción es capaz de hacerse cargo de su singularidad y apropiarse de lo transmitido para dar una nueva vuelta, que implica un cambio en la propia posición, una posibilidad de diálogo con las propias sujeciones, diálogo que se traduce en un obrar de otro modo respecto de las mismas (Aulagnier \& Castoriadis, 2010).

A partir de estas postulaciones, la formación entendida como el asumir una forma y transformarse (Barbier, 2017) es parte inmanente de la subjetividad. Aunque pueda impulsarse desde el mundo del trabajo, como es el caso de la formación docente o de cualquier tipo de formación profesional, involucra a todo el ser (Medina, 2006), no solo al sí mismo profesional (Abraham, 1987).

La transformación subjetiva tendrá lugar en el interjuego entre la formación como una oferta de propuestas desde el sistema, como dispositivo (Souto, 2016) mediatizado por sujetos formadores, y la construcción que de esa situación realiza cada individuo. Este entramado puede generar efectos de subjetivación o de desubjetivación (Bleichmar, 2005). Se constituirá en una instancia de subjetivación cuando el sujeto en formación sea capaz de "construir una experiencia”.

Rodulfo (2013), desde una lectura que combina el psicoanálisis posfreudiano de Winnicot con el deconstructivismo de Derrida, plantea que la capacidad de tener experiencias o experimentar es lo más valioso en un ser humano porque es lo que le permite sentirse existir, sentirse vivo, llegar a ser real. Esta capacidad implica, por un lado, el estar presente, conectado con el instante; por otro, el agenciamiento de lo vivido, esto es, el hacerse cargo del propio desear. Este estado se caracteriza por ir acompañado de espontaneidad, autenticidad, conexión con lo lúdico y la alegría como emoción predominante. Rodulfo (2013) vincula la posibilidad de experimentar con lo que él define como libertad en cuanto posibilidad de reinvención y la opone a lo que sería el reaccionar, actitud de respuesta ante una situación de violencia o un holding inapropiado. Es decir, el configurar una experiencia constituye una posibilidad que puede tanto promoverse como perjudicarse u obturarse.

Ante situaciones vividas como amenazantes, las personas reaccionamos poniendo en juego un sofisticado sistema de defensas, técnicas que el yo utiliza para reducir o suprimir, de forma más o menos consciente, aquello que es percibido como susceptible de poner en peligro la integridad propia o de causarle displacer o sufrimiento (Laplanche \& Pontalis, 2006). Al reaccionar perdemos la oportunidad de adueñarnos de nuestra experiencia, de recrearla, porque en lugar de permanecer ligados a nuestro desear, estamos defendiéndonos. La denegación, la evitación, la disociación, la proyección en el afuera de lo propio, el fantaseo alucinatorio, la constitución de un como si y la inhibición son algunas de ellas. La inhibición, dentro de estos mecanismos, actúa en tres planos: el de la acción, el del placer y el del deseo.

Estas cuestiones han sido abordadas también por los estudios realizados desde el campo de la psicodinámica del trabajo (Dejours, 2019). En esta línea se parte de la centralidad del trabajo para el despliegue de la subjetividad. Tomando el concepto de sublimación 
de Freud, como cambio del destino de la pulsión, de una meta sexual a una social, Dejours (2019) señala que el primer nivel de la sublimación es el del cuerpopriación que es intrasubjetivo. En el mundo del trabajo se produce un segundo nivel de sublimación que ocurre a través del reconocimiento del otro, es decir, que es intersubjetivo. Este reconocimiento puede tener lugar aun cuando el trabajo no esté puesto al servicio del bien común. Cuando además del reconocimiento aparece la ligazón con el bien común, estamos ante un tercer nivel de sublimación que mantiene el lazo entre el trabajo y el crecimiento individual y el trabajo y el desarrollo cultural a nivel colectivo. Cuando este tercer nivel de sublimación no tiene lugar, en cambio, y solo obtenemos reconocimiento, pero apoyamos actos que moralmente reprobamos, tiene lugar lo que Dejours (2019) denomina sufrimiento ético, que surge de la experiencia de la traición a nosotros mismos. Al traicionarnos, traicionamos también nuestros ideales.

Ante este sufrimiento, Dejours (2019) identificó cómo las defensas que el psicoanálisis había descrito se ponían en juego en el lugar del trabajo: una de ellas consiste en ocuparse, sobrecargando el aparato psíquico con un activismo frenético; otra forma implica aletargar o entumecer la capacidad de pensar o evitar los pensamientos vinculados al conflicto ético, anestesiándonos, de modo de no sentir culpa o vergüenza. Se trata en todos los casos de una renuncia o resignación frente a la presión productiva a cambio de algún beneficio, por ejemplo, ser aprobado o ser promovido, o a cambio de no ser agredido o castigado, una forma de trueque promovida por el miedo a alguna demostración de poder en la que se accede a hacer algo no aceptable para uno. Desde el punto de vista psicológico, este trueque constituye un perjuicio para el despliegue de la subjetividad en cuanto supone la pérdida de poder sobre los propios actos y la experimentación de la desesperanza y la desconexión con el propio deseo. De este modo, atenta de forma directa contra el narcisismo, contra la propia autoestima.

Una tercera mirada que se enfoca en los modos de sujeción — los mecanismos para gobernar la acción de otros - así como en los modos de resistencia a los mismos, completa un marco que nos permitirá enriquecer nuestro análisis (Ramírez-Zuluaga, 2015). Foucault (1991) en su última etapa intentó dar cuenta del sujeto al interior de las luchas contra las formas actuales de individuación y sujeción. Mostró cómo el sujeto, en cuanto engranaje, aun siendo blanco del poder, puede reproducirlo, pero también puede transformar lo que el mismo vehicula a través de la relación consigo mismo - lo que él llama gobierno de si - y fabricar otros modos de relacionarse (Foucault, 2000; Ramírez-Zuluaga, 2015). Particularmente, identifica dos tipos de prácticas de sí que dan cuenta de modos de resistir al poder: la subjetivación y la desubjetivación. La segunda forma —la desubjetivación- se refiere a la posibilidad de desprenderse de sí mismo para pensar de otro modo y devenir algo diferente con nuevas formas de relacionarse con otros. Foucault (2000) la relaciona con el movimiento que producen la escritura y la literatura, que favorecen formas de distanciamiento que habilitan la pluralidad de posiciones y la discontinuidad de las funciones (Blanchot, 2009).

Estos conceptos nos proporcionan una lente a través de la cual interpretar ciertas situaciones de nuestro estudio de caso con el fin de arrojar luz sobre la particular instancia de formación que la residencia significó para Francisca y para Yolanda.

\section{Método}

\subsection{Posicionamiento epistemológico, estrategias metodológicas y procedimiento seguido en el análisis de la información}

El trabajo forma parte del proyecto de investigación "La formación profesional: la residencia docente en el marco de la formación en las prácticas", dirigido por Marta Souto, con subsidio UBACYT, sede en el IICE, UBA, de 2014 a 2017. Epistemológicamente se encuadra en el paradigma de la complejidad (Morin, 1996), y desde una tradición hermenéutica y comprensiva, en el enfoque clínico, que supone como modalidad de construcción del conocimiento el abordaje en profundidad de casos singulares 
estudiados en terreno con el fin de lograr la comprensión a través de la elaboración inductiva de hipótesis interpretativas, el seguimiento de los procesos en su temporalidad y en su situación particular y la inclusión del propio investigador como sujeto en los fenómenos que estudia (Souto, 2010). Implica una relación intersubjetiva entre el investigador y un sujeto social como objeto de conocimiento, que busca la comunicación no solo consciente sino inconsciente con el otro, de lo manifiesto y lo latente. Involucra también el compromiso ético y el respeto hacia el otro, que, entre otras cosas, se manifiesta en el esfuerzo de co-construcción de sentido en el conocimiento que se genera. Este compromiso implica, asimismo, poner en análisis la propia implicación del investigador con el caso que analiza.

Si bien el proyecto original consideró como unidades de análisis a la institución formadora, a las distintas instancias del dispositivo de residencia, a los profesores de residencia, a la escuela y sus actores, y a los residentes, el presente trabajo toma únicamente la última de estas unidades: los residentes.

Con el objetivo de contribuir al conocimiento de algunas de las posibilidades, así como las limitaciones que brinda la residencia como instancia formativa y de los diferentes modos de vivenciarla, en particular, se seleccionaron como estudio de caso dos residentes, a quienes se acompañó a lo largo de su último año de formación en un Instituto Superior de Formación Docente de dependencia oficial, público, de la provincia de Buenos Aires, y durante su estadía en la escuela en la que realizaron sus prácticas.

La estrategia metodológica combina y triangula los datos de cada docente obtenidos a partir de:

- entrevistas en profundidad (2) (antes de comenzar y al finalizar).

- observaciones y notas de campo tomadas durante las 4 clases de cada residente en el marco de la residencia.

- entrevistas breves cara a cara y toma de notas en situaciones de soliloquio durante la estadía en la escuela esos 4 días.
Siguiendo una lógica cualitativa, se construyeron $\mathrm{ca}$ tegorías a partir de los datos de manera inductiva. Se empleó el análisis de contenido (Krippendorf, 1990) y el análisis del discurso (García-Negroni \& Tordecillas, 2001) para las entrevistas y el análisis didáctico multirreferenciado (Souto, 2004) para las situaciones de clase. Los análisis provenientes de las distintas fuentes respetan su heterogeneidad y se triangulan posteriormente. Luego de esta triangulación se elaboraron hipótesis clínicas (Souto, 2010). La formulación de estas hipótesis, en el caso considerado, implicó una reconstrucción del proceso vivido por las residentes, desde una mirada interpretativa del investigador, a partir de los indicios recogidos. Se complementó este análisis con conceptos provenientes de las teorías psicoanalítica (Bleichmar, 2005; Rodulfo, 2013), de la psicodinámica del trabajo (Dejours, 2019) y foucaultiana (Foucault, 1991, 2000), desarrollados en el apartado anterior.

\subsection{Dispositivo de residencia}

Se compone de el taller de residencia a cargo de un/a profesor/a de residencia; los ateneos de matemática, prácticas del lenguaje y la literatura, ciencias sociales y ciencias naturales, cada uno de ellos a cargo de un profesor especialista en el área disciplinar respectiva, y el Taller Integrador Interdisciplinario (TAIN) en el que una vez por mes se abordan temáticas abiertas consideradas necesarias. Todas estas instancias están pensadas en función del núcleo de la formación en el campo de las prácticas, que es la residencia propiamente dicha. Consiste en prácticas en terreno que duran seis semanas en cada cuatrimestre, a realizar en dos cursos de distinto ciclo de la escuela primaria y que abarcan el conjunto conformado por observaciones del curso (2 semanas), prácticas aisladas (1 semana) y luego la secuencia de prácticas (3 semanas seguidas) en que la residente toma a cargo todas las clases. Esta secuencia se retoma a posteriori en las clases del taller y los ateneos para generar una reflexión sobre las prácticas. Este dispositivo involucra como formadores en el instituto de formación: al profesor de residencia y a los profesores de las áreas. En la escuela en la que se hacen las prácticas, involucra al maestro de grado (que cumple la función de maestro coformador en este caso) y, a veces, al director. 


\subsection{Las residentes, docentes en formación: Francisca y Yolanda}

Ambas inician su residencia en el 4to. año del Profesorado de Formación Docente en el año 2014 en un Instituto Superior de Buenos Aires. Ambas han elegido tanto la escuela primaria en la que realizarán las prácticas, como a la profesora de residencias que las supervisará. Ambas han accedido voluntariamente a participar en la investigación y se les ha garantizado el anonimato, de allí el empleo de un pseudónimo.

Francisca tiene 28 años, y la residencia constituye su primera experiencia al frente del aula. Yolanda tiene 26 años. Tiene una formación previa en otra carrera terciaria. Intentó ejercer, pero "no funcionó". A partir de esa experiencia descubre su interés por los otros y por "brindar ayuda", que parece estar en la base de su elección de la carrera docente. Lleva un ańo realizando suplencias en escuelas, de modo que la residencia no es su primera experiencia docente.

\section{Resultados}

\subsection{El deseo de enseñar, las defensas y la configuración de una experiencia en una situación compleja de formación}

\subsubsection{Francisca en la residencia}

Antes del inicio de las prácticas, conviven en Francisca sentimientos ambivalentes: de ansiedad frente a cumplir con la planificación exigida por el instituto — "Estoy como loca con el proyecto integrado"de miedo al fracaso - "Ay, me va a ir mal" — en lo que experimenta como una situación de prueba en la que tiene que cumplir con demandas de diferente índole, contrapuesto a cierta sensación de seguridad en cuanto a poder ejercer su rol — "Muchas ganas de trabajar en el estado y seguridad de que puedo hacer muchas cosas buenas"-, junto con altas expectativas - "Este es un año reimportante" - y el deseo de hacer la experiencia — "Desde que empecé estoy esperando que llegue este momento"-, y una representación acerca de los niños como aquello de la docencia que la convoca — "Los nenes son una mina de oro, podés hacer y deshacer. Quieren aprender"-. Aun ante la inseguridad que sufre asociada al miedo, al posible fracaso y al rechazo - "Prefiero que me lo digan ellas [las profesoras de prácticas] y no una directora que por ahí me echa por eso"-, está conectada con su deseo de ser maestra - "La verdad, no sé por qué dije que iba a estudiar para maestra, ahora me gusta más que antes" - y con su capacidad de "hacer muchas cosas buenas".

En la primera semana de prácticas, de a poco comienza a escindir y separar aspectos positivos en ella y a proyectar los negativos en la escuela. Así habla de la primera devolución de la profesora de prácticas: "Me dijo que me ve muy bien, muy segura, muy bien parada". Y expresa respecto a sus primeras experiencias al frente de una clase: "Lo que planificás lo llevás y te das cuenta de lo que es estar en el aula, que me encanta. Con los chicos me llevo bárbaro. Eso me da mucha confianza". Pero al mismo tiempo comienza a encontrar aspectos negativos en los modos de trabajo de la escuela: "Es que ellos están acostumbrados a trabajar todo muy por arriba. Se los dan una sola vez y después nunca más”. Se instala así la base de un conflicto ambivalente - en el que intervienen motivaciones incompatibles - que persistirá a lo largo de toda la residencia para ella.

Con respecto al vínculo con la directora y las maestras, Francisca da cuenta de malestar y tensión desde el primer día de llegada a la escuela, al encontrar, junto con otras residentes, que no las esperaban. La directora no había avisado al personal que ellas asistirían. "La directora nos aceptó porque le da puntaje a la escuela, pero no sé para qué". "Me encajaron un proyecto de la escuela de matemática... me dicen que es cosa de la directora". "Me cargaron con la feria de ciencias, tuve que hacer un cartel para la puerta de la otra residente. Esto no me correspondía a mí... me cargan de cosas. Así que le hice uno pedorro". Establece un vínculo negativo con la escuela y su personal. "Pedorro" en lunfardo (jerga argentina) significa de mala calidad. Francisca percibe falta de receptividad de la escuela y se muestra muy crítica hacia los miembros de la institución. Los describe como autoritarios, indiferentes, interesados. Además, se 


\section{MANRIQUE}

Formación docente en la residencia. ¿Experiencia subjetivante?

percibe como blanco de actos injustos y sobrecargada de funciones que según ella no le corresponden. Aun con enojo y disgusto, se resigna, sin embargo, a cumplirlas. Vive a la directora como figura amenazante — "Por ahí me echa"—, temor asociado a la experiencia de otra residente cuya práctica fue suspendida, con la que se identifica.

Con la maestra coformadora, el vínculo parece tener componentes similares. Francisca se siente observa$\mathrm{da}$, controlada, incómoda en la clase frente a sus interrupciones: "No le debe gustar mucho que yo este acá. Todo el tiempo se mete. En realidad, yo quiero que ella se vaya y me deje un poco el camino libre".

Frente a estas situaciones, la relación con la profesora de residencia es significativa, es ella quien inicialmente apuntala psíquicamente a Francisca, le da seguridad y apoyo, constituyéndose en una figura protectora - "Ella nos contiene, traemos planificaciones y vemos las dudas, cómo manejo esto o aquello... Si mandás mail te responde enseguida”-

Sin embargo, este vínculo con la profesora de residencia que la reaseguraba en un contexto que percibía como hostil va transformándose a lo largo del proceso, a partir de diferentes situaciones en que Francisca experimenta impotencia y frustración. Mientras ella da la clase, la maestra coformadora le muestra a la profesora de residencia un afiche hecho por Francisca y le señala errores que no le había marcado a Francisca. Posteriormente, la profesora de residencia le pide disculpas a Francisca — "No sabía cómo frenarla" - . En otra oportunidad, "la directora viene a verme y me dice que mi clase no tiene nada que ver con el diseño curricular. Nadie hizo nada para que la directora no se meta”.

A medida que transita la experiencia, Francisca va pasando de la ilusión a la desilusión. Los cambios en la relación con la profesora de residencia forman parte de este pasaje, significando una pérdida de confianza en la única figura que le proveía seguridad. De ella dice: "No se enfrenta a las autoridades de la escuela, no defiende el espacio, se deja manejar por la directora". Se percibe sola frente al contexto hostil que la escuela representa.
Con respecto al dictado de las clases, Francisca se enfrenta a una situación que experimenta de modo paradojal: o responde a lo que le piden en la escuela y es "tradicional" o responde a lo aprendido en el instituto y a sus ideales y deseos y es "constructivista”. Surgen en ella sentimientos de impotencia — "Sé que caí a veces en eso y me lo critico, pero a veces es imposible ir contra lo tradicional"-y frustración, debido a la imposibilidad de implementar aquello que le han enseñado en el instituto y que ella deseaba poder poner en práctica — "Hubiera querido ser más constructivista"-, a la vez que se siente coartada en su espontaneidad y libertad de acción — "Por favor, dame el título, así puedo ser yo”- - Su malestar va creciendo a lo largo del proceso — "Entonces, ¿con quién tenemos que cumplir? Con los gustos y caprichos de todo el mundo. No hay una línea. No podemos ser naturales nosotras porque estamos vigiladas y controladas por todos lados"-.

En efecto, en el dictado de sus clases la observación muestra cómo recurre a modos de intervención que criticó en las maestras y en el instituto como tradicionales. Desde lo social, sostiene un trato cada vez más autoritario con los alumnos y acentúa diferencias discriminatorias; desde lo instrumental, no logra un manejo ni dominio sobre el grupo y las propuestas de trabajo se vuelven cada vez más "tradicionales": escribir en el cuaderno, copiar, responder cuestionarios.

El miedo es la emoción que predomina: miedo a la maestra coformadora, a la profesora de residencia, a la directora - "La directora me preguntó si iba a estar toda la semana, como diciéndome: ¡Mirá que vas a estar vigilada! Si me llega a levantar la residencia, ¿sabés el quilombo que le armo?”- El sentimiento de ser controlada por todos aumenta sus ansiedades persecutorias. "Te tenés que acomodar a su estilo porque, si no, es muy chocante para ella. Entonces caigo en lo tradicional y dejo conforme a todo el mundo".

Desde el punto de vista psíquico, ante lo que experimenta como amenazas contra su integridad yoica, un conjunto de defensas parece instrumentarse para preservarla. Sus defensas incluyen la evitación de las 
situaciones de control (estrategia utilizada para no ser observada por profesores que la van a observar) y la evitación de la residencia misma (en tanto desea que termine rápido); el desvalorizar la experiencia de formación, transformándola en un objeto parcial negativo - "Para mí, la residencia es algo jodido"y la proyección sobre los otros: escuela, instituto, profesora de residencia, maestra coformadora, de los aspectos negativos, mientras que ella se desentiende de su propia responsabilidad en las situaciones. Por ejemplo, frente a una devolución de la profesora de residencia en la que se señalan aspectos a modificar se justifica, busca equivocaciones en los otros y deposita en el afuera la fuente de los problemas. En pocas oportunidades reconoce que hay algo que ella pueda mejorar, cambiar o que no haya salido bien. Se autopercibe como víctima de la situación. La posibilidad de reflexión, de toma de conciencia y de transformación se ve afectada por estos mecanismos defensivos.

Frente a esta situación en la que predomina la sensación de ser evaluada - "Tenés que cumplir las expectativas de todo el mundo y con las tuyas para no traicionarte. Ahí está el problema, con quién cumplimos"- y el sentimiento de miedo ante la posibilidad de desaprobar, Francisca inhibe su actitud deseante y resigna sus ideales adoptando una lógica de cumplimiento formal de la tarea y de aprobación, en pos de una adaptación a las condiciones áulicas que siente impuestas, y con las que no coincide. De las demandas que identifica, Francisca privilegia aquellas que la alejan de sí misma, de su ideal y de sus creencias, que la hacen "traicionarse", en sus propias palabras. $\mathrm{Su}$ actuación en la residencia es vivida como un "como si”, una ficción, fabricada para sobrevivir en ese contexto - "No fui yo en el aula. Fui un producto, resultado, de todas las cuestiones internas que hubo"-. Calificada por ella como "mala experiencia", la residencia, bajo la forma de una práctica enajenada, que no le pertenece, que es una escena armada para otros, parece ser la solución de compromiso que encuentra en este contexto vivido como amenazante. Como correlato, el aprendizaje propio de su formación en las prácticas y el desarrollo de su capacidad reflexiva quedan reducidos. - "Antes yo tenía reganas de cambiar, pero cuando estás acá, decís: ¿En tres semanas, cuánto voy a poder hacer? Entonces voy a dar las clases para aprobar y listo"- En la evitación del sufrimiento presente pierde la posibilidad de ponerse en análisis ella misma y posterga el aprendizaje hacia el futuro - "Cuando tenga mi grado voy a tener la libertad de aplicar cosas más personales, no ya del profesorado, ni de Piaget, ni de toda esa cagada de psicología evolutiva”-

\subsubsection{Yolanda en la residencia}

Inicia su proceso señalando que no se está "topando con algo nuevo", porque ya lleva un año dando clase, lo cual parece brindarle cierta tranquilidad. Remarca que las devoluciones que pueda recibir no van a frustrarla, a desanimarla; por el contrario, se convierten en un aporte para su formación y para su práctica actual.

Desde su experiencia docente actual, Yolanda contrasta y evalúa lo que la maestra coformadora le propone, lo que la ubica más en una posición de par que de novata. Su planificación se separa de la propuesta del manual que le dio la maestra coformadora y contempla conocimientos previos de los estudiantes. El encontrarse trabajando también le permite tomar cierta distancia de lo que le han enseńado en el instituto, lo que le posibilita evaluar críticamente las situaciones concretas con sus múltiples aspectos — "Nadie te enseńa qué contestarle a un chico cuando trata mal a otro"-.

A pesar de estar trabajando ya, Yolanda no parece haber perdido su posición de aprendiz, de sujeto en formación - "Nunca voy a terminar de formarme"- Valora la residencia como situación única e irrepetible — "No voy a tener nunca más la mirada de otro docente en el aula diciéndome qué es lo que estoy haciendo bien y qué no"-

En relación con los vínculos, la maestra coformadora la recibe con un mensaje que denota desconfianza - "Me dijo que tuvo residentes y que los chicos no entendieron nada"-, pero ella no parece afectada por esto — "Bueno, no sé: esa es su historia”Sobre su profesora de residencia da una imagen de acompañamiento, presencia y complicidad. Hacia 
los alumnos, tiene una mirada muy focalizada al trabajo: los ve participativos y haciendo chistes, no parecen generarle preocupaciones vinculadas a la gestión de la clase.

En la primera clase que dicta se presentan una serie de incidentes que dan cuenta de dificultades en la distribución de roles entre ella y la maestra coformadora. La maestra coformadora reparte evaluaciones sin permitir que Yolanda comience, da permisos o no a los alumnos para salir del aula, habla con otra maestra en voz alta dentro del aula durante la clase. Sobre el final, toma directamente la dirección de la clase cuando Yolanda se encuentra dando la tarea, para modificar lo pedido, contradiciéndola — "(Yolanda): Pueden hacer lo de sociales el martes o el miércoles porque recién el jueves lo vamos a ver. (Maestra coformadora, interrumpiendo): Lo hacen todo el fin de semana porque yo lo tengo que ver. (A Yolanda): Vos no los conocés. Se te duermen, ellos. (Al grupo): Por eso les aclaro, porque ella es buena y no los conoce. (Yolanda mira a la observadora incómoda y se retira del aula antes que los estudiantes)"-. Estas intervenciones resultan disruptivas, inhibitorias y descalificadoras del rol de Yolanda. Son un indicio de la dificultad de la maestra coformadora para aceptar su rol no participante y ceder la dirección de la clase. $\mathrm{Al}$ reafirmar su posición como quien manda, propone, en cambio, una relación de rivalidad y competencia con la residente.

Ante esta situación, Yolanda recurre a su profesora de residencia, quien a su vez interviene conversando el tema con la directora de la escuela. Esto genera un mayor conflicto entre Yolanda y la maestra coformadora, quien se queja ante Yolanda — "Ponés en jaque mi trabajo"-. Sin embargo, este conflicto no perjudica el vínculo entre ambas, sino que Yolanda empatiza con la maestra coformadora, como se observa en el fragmento:

— "No estuvo bueno, a mí tampoco me pareció la forma. Raro, mientras estaba haciendo la residencia, tuve practicantes en mi grado, en que yo era la docente. Entonces, fue muy fuerte, y no solo que me di cuenta que no era personal, sino que me di cuenta: es que cada uno... tiene un lazo particular con el grado, con tu grado, 'el mío', ¿viste? Y por el otro lado, me parece que también la forma en que uno cree que se debe dar la enseńanza, cada uno tiene sus convicciones, sus teorías y sus formas, ¿no? Y me parece que esas dos cosas mezcladas en un ambiente, en un aula, con alguien externo, me parece que es chocante. Porque yo no puedo juzgarla a ella, ni como enseńa, ni cómo se dirige a... o sea, nada..."-

Yolanda problematiza la situación mirándola desde su lugar y también desde el lugar de la docente - "Empecé a pensar esto de que no era nada personal, que tenía que ver con ella, con su aula y sus alumnos, y con la forma en que quería que ellos aprendan y bueno, nada, y yo estaba queriendo recibirme"-.

Esta actitud comprensiva que le permite distinguir diferentes posicionamientos con intencionalidades diversas parece favorecida por la condición de docente en ejercicio que atraviesa Yolanda.

A partir de esta situación, se observa cómo en el dictado de las sucesivas clases Yolanda gana firmeza y se hace su lugar, y paralelamente la maestra coformadora deja de avanzar sobre su rol y da lugar a ese movimiento. Por un lado, las intervenciones de la maestra coformadora disminuyen —en una de las clases, por ejemplo, permanece afuera conversando con un padre, tarea que los residentes no están habilitados a realizar, mientras Yolanda da la clase-. Durante las otras dos clases se observa a Yolanda a cargo de la clase y a la maestra coformadora cumpliendo un rol complementario de respaldo. Cuando interviene, su rol es más de acompañamiento a la residente que de rivalidad. Se evidencia cooperación por parte de ambas, por ejemplo, en el acuerdo ante el envío de una nota en el cuaderno de comunicaciones, para lo cual se consultan entre ellas qué poner. Esto refleja un cambio respecto a la primera clase observada. El vínculo entre ambas, en lugar de haberse perjudicado por lo ocurrido, parece haber tomado la forma de un vínculo cooperativo.

Al finalizar la residencia queda en Yolanda una serie de dudas acerca de cómo será afectada por la práctica de la docencia en sus convicciones más profundas: 
- "Lo que me deja pensando es intentar que este enfoque del profesorado, esta forma de dar clases, eh, mantenerla a lo largo del tiempo. Cuando terminás la carrera estás en el auge de querer ser la mejor maestra que te enseñaron ser, ¿no? Pero, el miedo me parece de muchos, no solo mío, es esto de que después el sistema te coma o la cotidianeidad y termine siendo la maestra que en el profesorado critiqué. Eso es, pero bueno, es muy difícil”-

Se manifiesta un posicionamiento ético en Yolanda y, a la vez, una exigencia profesional (querer ser la mejor maestra que te enseñaron) y el temor de no poder sostenerlo — "que el sistema te coma"-. Yolanda culmina la residencia con reflexiones sobre su propio posicionamiento docente a partir de la experiencia y reafirmando sus ideales con relación al futuro.

\section{Discusión y conclusiones}

El trabajo parte de la pregunta acerca de las oportunidades que brinda la instancia de residencia docente para el desarrollo de la subjetividad y del sí mismo profesional. Se describen y analizan dos recorridos diferentes por la residencia a lo largo de un semestre, haciendo foco en el modo en que, en ellos, las residentes lidian con las demandas que se les presentan.

En los dos recorridos que hemos descrito y analizado se puede apreciar la complejidad del escenario de la residencia —demandas contradictorias, vertiginosidad de los tiempos, multiplicidad de actores participantes: directivos, docentes coformadores, docentes del instituto, profesora de residencia, estudiantes-. En ambos se advierte la cualidad tensionante de la situación en cuanto pasaje de pruebas - la residencia es una instancia de formación, pero también de acreditación, y está atravesada por la lógica evaluativa, tal como se ha descrito en otros trabajos (Souto, 2016)—.

En ambas situaciones se observan dificultades en el proceso, asociadas sobre todo al vínculo con la maestra coformadora del curso. Sin embargo, los conflictos dan lugar a modos distintos de posicionarse frente a ellos y de resolverlos, que y de resolver, que afectan las oportunidades de desarrollo subjetivo de las residentes. En ambas, la actividad deseante constituye inicialmente un motor; en un caso se reafirma a lo largo del proceso, en el otro se resigna. Veamos cada caso a la luz de conceptos psicoanalíticos y foucaultianos.

En el caso de Francisca, podríamos interpretar que tiene lugar lo que Dejours (2019) identifica como "sufrimiento ético", la experiencia de la traición a uno mismo y a los propios ideales, que sucede cuando opera el segundo nivel de sublimación —el reconocimiento del otroque moralmente reprobamos. La actitud posterior de Francisca de "entumecimiento" que no le permite ver críticamente que su propio comportamiento hacia los estudiantes en el aula reproduce lo que ella misma criticaba. Esto podría ser interpretado, en este marco, como parte de una estrategia defensiva al servicio de la evitación de un posible castigo — por ejemplo, la desaprobación-.

Desde el punto de vista psíquico podríamos pensar, siguiendo a este autor, que esta claudicación de sus propios ideales implica la pérdida de poder sobre los propios actos y constituye un perjuicio para el despliegue de su subjetividad, ya que atenta contra la propia autoestima. Francisca habría trocado su silencio antes situaciones que no comparte por la aprobación de su residencia. En esta transacción ha puesto en juego los mecanismos que el autor también ha descripto, básicamente la insensibilización. La renuncia a su deseo, acompañada del sentimiento de hastío y desesperanza — su deseo de huir de la situación de residencia-, así como la perdida de amor propio — "No fui yo [...] Me hubiera gustado ser más constructivista”-, podrían ser pensadas como el correlato de este "trueque".

La residencia — “una mala experiencia” en palabras de Francisca - se ha constituido en una instancia que según Bleichmar (2005) se designaría como desubjetivante, en cuanto no favorece la inscripción psíquica de la experiencia, el agenciamiento de la misma. Desde la teoría de Rodulfo (2013) podríamos hipotetizar que ante la situación de violencia que su entorno le ofrece y el holding inadecuado de sus figuras 
de referencia, Francisca, en función de su autopreservación yoica (Bleichmar, 2005), habría puesto en juego el mecanismo de la reacción. La reacción en ella toma la particular forma de la inhibición. Inhibe su espontaneidad, su actividad deseante, su desear. Pierde así la posibilidad de construir una experiencia —no se constituye en agente de la misma, sino que resigna esa posición de agente-.

En el caso de Yolanda, en cambio, las dificultades iniciales con la maestra coformadora y lo que la propia residente consideró una intervención desafortunada de su profesora de residencias no le impidieron poner en juego su capacidad de autoafirmación de sí —la emergencia de la palabra propia, de su propia singularidad-y el sostener su agencia, que implica hacerse cargo de su desear y construir los propios proyectos identificatorios (Aulagnier \& Castoriadis, 2010). Siguiendo a Rodulfo (2013), podríamos pensar que ella ha podido construir una experiencia de la vivencia que, por tanto, deja en ella una marca subjetivante (Bleichmar, 2005). Se evidencia en la transformación del vínculo con la coformadora y en la posibilidad de Yolanda de extraer de la situación elementos que le permitieron analizar y construir su rol profesional de modo autónomo. También es evidente en el correlato emocional: Yolanda experimenta alegría y entusiasmo, mientras que en Francisca prevalecía la desesperanza, el enojo, la frustración y la impotencia.

Si pensamos la situación desde la óptica del poder (Foucault, 1991, 2000), el caso de Yolanda pone en evidencia la posibilidad de reconducción del poder a través de la relación consigo misma o de gobierno de sí. Tomando la idea de Foucault (2000), podríamos pensar que es a partir de esta posibilidad que Yolanda en su calidad de engranaje habría sido capaz de transformarse y de fabricar otros modos de relacionarse. Yolanda parecería haber hecho uso de la práctica de sí que Foucault denominó "desubjetivante", que consiste en desprenderse de sí misma para pensar de otro modo y devenir algo diferente con nuevas formas de relacionarse con otros. En su modo de identificarse con la maestra coformadora y de redefinir su vínculo con ella - momento bisagra de la redefinición de toda su situación-, algo de esto parece haberse puesto en juego. En el caso de Yolanda, este movimiento de distanciamiento que habilita la pluralidad de posiciones y la discontinuidad de las funciones (Blanchot, 2009) parece haber sido facilitado por las condiciones de su experiencia: por el hecho de ocupar efectivamente posiciones diferentes - como residente y como docente-, simultáneamente en dos lugares distintos.

Por todo lo mostrado hasta aquí, se puede concluir que el trabajo constituye una evidencia empírica de efectos subjetivantes y desubjetivantes (Bleichmar, 2005) que la residencia puede tener en docentes en formación.

Dos situaciones se presentan. Ambas constituyen experiencias de formación en cuanto que en ambas se observa trasformación. Las residentes salen diferentes de como entraron: se afirman ciertas tendencias, se niegan otras. Sin embargo, las dos proporcionan oportunidades diferenciadas para el desarrollo de la subjetividad y del sí mismo profesional (Abraham, 1987) de las futuras docentes.

Estos resultados nos hablan, por una parte, de la relevancia de la residencia como instancia de construcción de la subjetividad de las docentes y de su sí mismo profesional y, por otra, del grado de heterogeneidad de las experiencias vivenciadas, que se revela en cómo en cada situación, y desde diferentes rasgos de personalidad y modos de posicionarse, las situaciones con demandas complejas son reeditadas y se convierten en posibilidades de subjetivación o desubjetivación, de construcción o no de una experiencia, de agenciamiento o de inhibición de la propia actividad deseante.

Los resultados también muestran, desde la óptica del poder (Foucault, 1991, 2000), cómo se puede dar el juego de sumisión o de resistencia en el vínculo de los sujetos con el sistema.

A partir de estos resultados se pone de manifiesto la importancia de salvaguardar la experiencia de formación en cada residente desde la propuesta de formación y desde el sistema para que esta posibilidad no quede librada al azar o a la posibilidad subjetiva 
y singular de cada docente en formación. Sin duda, como formadores nos cabe una responsabilidad en este sentido en cuanto al modo que asume el acompañamiento en una instancia tan delicada. La función continente, el holding, adquiere prevalencia para propiciar efectos subjetivantes.

Este conocimiento nos enfrenta con el desafío de diseñar en la formación instancias y modos de acompañamiento que contemplen toda esta gama de posibilidades a las que la singularidad del devenir subjetivo da lugar, para que la residencia pueda constituirse en dispositivo de reflexión, tal como Branda (2018) o Steiman (2018) lo describen. Desde el punto de vista psíquico, se trata de una apuesta a la formación como promoción de la singularidad que no se propone ni la adaptación social en función de ciertos ideales, ni la abolición del conflicto como meta, sino que se basa en sostener la emergencia de la palabra propia a partir de la habilitación de la actividad deseante de cada docente en formación.

El desafío vale la pena. Y el riesgo de no encararlo implica la posibilidad de perder oportunidades de subjetivación únicas y muy valiosas para las futuras docentes y de dejar, en quienes sufren las consecuencias, marcas que pueden obturar su desarrollo profesional.

\section{Agradecimientos y reconocimientos}

La investigación fue realizada con el subsidio UBACYT 2014-2017 - 01/Q785 y con fondos del CONICET, donde me desempeño en calidad de investigadora adjunta.

Agradezco las discusiones y recomendaciones de las licenciadas María Clara Manrique y Graciela Manrique.

\section{Referencias}

Abraham, A. (1987). El mundo interior de los enseñantes. Buenos Aires: Gedisa.
Ardoino, J. (2005). Pensar la educación desde una mirada epistemológica. Buenos Aires: Ediciones Novedades Educativas.

Aulagnier, P., \& Castoriadis, C. (2010). La violencia de la interpretación. Amorrortu.

Barbier, J. M. (1999). Prácticas de formación. Evaluación y análisis. Formación de Formadores. Buenos Aires: Ediciones Novedades Educativas.

Barbier, J. M. (2017, july). La transformation continue par les sujets des rapports qu'ils entretiennent avec/entre les composants de leur activité. Conference papier. https://r.issu.edu.do/l?l=101919VG

Bleichmar, S. (2005). La subjetividad en riesgo. Buenos Aires: Topía.

Blanchot, B. (2009). Michel Foucault tal como lo imagino. Madrid: Arena Libros.

Branda, S. A. (2018). El lugar de la práctica en la formación docente inicial. Las residencias como dispositivo de reflexión. Archivos de Ciencias de la Educación, 12(13), e044, https://doi.org/10.24215/23468866E044

Dejours, C. (2019). El sufrimiento en el trabajo. Buenos Aires: Topía.

Ferry, G. (1997). Pedagogía de la formación. Buenos Aires: Ediciones Novedades Educativas.

García-Negroni, M. M., \& Tordecillas, M. (2001). La enunciación en la lengua. De la deixis a la polifonía. Buenos Aires: Gredos.

Krippendorf, K. (1990). Método de análisis de contenido. Teoría y práctica. Buenos Aires: Paidós.

Foucault, M. (1991). El sujeto y el poder. Bogotá: Ediciones Carpe Diem.

Foucault, M. (2000). Un diálogo sobre el poder y otras conversaciones. Madrid: Alianza.

Laplanche, J., \& Pontalis, J-B. (2006). Diccionario de psicoanálisis. Buenos Aires: Paidós.

Medina, J. (2006). El malestar en la pedagogía. El acto 
de educar desde otra identidad docente. Buenos Aires: Noveduc.

Morin, E. (1996). Introducción al pensamiento complejo. Buenos Aires: Gedisa.

Najmanovich, D. (2011). El juego de los vinculos. Subjetividad y redes: figuras en la mutación. Buenos Aires: Biblos.

Ramírez-Zuluaga, L. A. (2015). El sujeto en los juegos del poder. Subjetivación y desubjetivación desde Foucault. Revista de Psicología Universidad de Antioquia, 7(2), 133-146. https://cutt.ly/9r4C3TT

Rodulfo, R. (2013). Andamios del psicoanálisis. Lenguaje vivo y lenguaje muerto en las teorias psicoanaliticas. Buenos Aires: Paidós.

Schön, D. (1998). El profesional reflexivo. ¿Cómo piensan los profesionales cuando actúan? Buenos Aires: Paidós.
Souto, M. (1999). Grupos y dispositivos de formación. Serie Formación de Formadores, 10. Buenos Aires: Ediciones Novedades Educativas.

Souto, M. (2004). La formación de docentes en el análisis multirreferenciado de clases. Revista Educación, Lenguaje y Sociedad, 2(2), 5-18. https://cutt.ly/Qr4Vbns

Souto, M. (2010). La investigación clínica en Ciencias de la Educación. Revista del IICE, 17(29), 5774. https://cutt.ly/or4VjYx

Souto, M. (2016). Algunos problemas y sentidos que la formación en la residencia plantea. Revista del CISEN. Tramas/Maepova, 4(2), 49-64. https://cutt.ly/Cr4Vebb

Steiman, J. (2018). Las prácticas de enseñanza -en análisis desde una Didáctica reflexiva-. Buenos Aires: Miño y Dávila.

\section{CÓMO CITAR:}

Manrique, M. S. (2021). Formación docente en la residencia. ¿Experiencia subjetivante?. RECIE. Revista Caribeña de Investigación Educativa, 5(1), 73-85. https://doi. org/10.32541/recie.2021.v5i1.pp73-85 